\title{
Two-step milling on the carbonyl iron particles and optimizing on the composite
}

\author{
absorption \\ Yonggang $\mathrm{Xu}^{1 *}$, Liming Yuan ${ }^{1}$, Xiaobing Wang ${ }^{1}$, Deyuan Zhang ${ }^{2}$ \\ 1. Science and Technology on Electromagnetic Scattering Laboratory, Shanghai, 200438, China \\ 2. Bionic and Micro/Nano/Bio Manufacturing Technology Research Center, School of Mechanical Engineering and \\ Automation, Beihang University, Beijing, 100191, China
}

\begin{abstract}
The flaky carbonyl iron particles (CIPs) were prepared using a two-step milling process. The surface morphology was characterized by the scanning electron microscopy, the static magnetic property was evaluated on a vibrating sample magnetometer and X-ray diffraction (XRD) patterns were done to analyze the particle crystal grain structure. The complex permittivity and permeability were measured using a vector network analyzer in the frequency range of $2-18 \mathrm{GHz}$. Then Hermite interpolation based on the calculated scattering parameters of the tested composite was used to derive the permittivity and permeability of the composite with random volume content. The results showed that the saturation magnetization value of the flaky CIPs decreased as the CIPs was changed to the flakes by high and low speeding milling. The diffraction peaks of the single $\alpha$-Fe existed in the XRD pattern of CIPs, and the characteristic peaks was broad and the intensity of the diffraction pattern was lower as the high-speeding milling time increased. The sample H2L20 had the largest particle size, the average diameter was $8.64 \mu \mathrm{m}$, the thickness was $0.59 \mu \mathrm{m}$ according to the fitted aspect ratio 14.65. The derived permittivity and permeability using the Hermite interpolation was accurate compared with the tested result, the deviation was about $0.39+\mathrm{j} 0.45$ and $2.5+\mathrm{j} 0.51$. Finally, the genetic algorithm was used to optimize the thickness of the CIPs composite of a wide absorbing band of 8-18 GHz. The optimized reflection loss (RL) result showed that the absorbing composites with thickness $1.47 \mathrm{~mm}$ had an excellent absorbing property $(\mathrm{RL}<-10 \mathrm{~dB})$ in $8-18 \mathrm{GHz}$.
\end{abstract}

Keywords: A.composite material; A. metals and alloys; B. mechanochemical; D. magnetic measurements

\section{Introduction}

Radar absorbing materials (RAM) have been widely used in the military applications and the civil

* Corresponding author: Yonggang Xu

E-mail: xuyonggang221@163.com 
aspects such as the stealth coatings, the absorbing patch on the phone, et al [1-3]. Recently, carbonyl iron particles (CIPs) are widely used due to the micrometer size, the large value of the saturation magnetization and the high Snoek's limit at gigahertz frequency. The saturation magnetization and the Snoek's limit could be described by the below equation $[4,5]:\left(\mu_{s}-1\right) f_{r}=\gamma M_{s} /(3 \pi), \gamma$ denotes the gyromagnetic ratio and $M_{s}$ is the saturation magnetization, $\mu_{s}$ is the static permeability and $f_{r}$ is the resonance frequency. Both $\mu_{s}$ and $f_{r}$ could not be increased at the same time, therefore, the spherical CIPs had a limitation in fabricating excellent absorbing materials with thin thickness in the wide frequency. For example, the reflection loss (RL) of composites added 55vol\% CIPs just reached to $-3 \mathrm{~dB}$ at $2 \mathrm{GHz}$ with $1 \mathrm{~mm}$ thickness [6], $-5 \mathrm{~dB}$ at $4 \mathrm{GHz}$ with $1 \mathrm{~mm}$ thickness as $93 \mathrm{wt} \%$ CIPs added [7]. As CIPs were shaped to the flakes, the existing easy-plane anisotropy supports a solution on exceeding the Snoek's limit. The parameters $\mu_{s}$ and $f_{r}$ for the easy-plane anisotropy material can be obtained [8, 9]: $\left(\mu_{s}-1\right) f_{r}=\gamma M_{s} \sqrt{H_{h a} / H_{e a}} /(3 \pi)$, where $H_{h a}$ and $H_{e a}$ denote the effective anisotropy field when the magnetization deviates from the easy axis in the hard plane and in the easy plane respectively. As a result, the permeability of composites added the same volume content flaky CIPs would increase. The results are attributed to the reduction of the eddy current loss, the orientation of the magnetic moment and the space-charge polarization with the shape change from spherical powders to thin flake particles [10]. The mechanical milling process became an economic process on converting the spherical shape to the flaky shape. Previous research had shown that thin the flaky CIPs could be obtained by high speeding milling on the spherical powders $[6,10,11]$, and the milling process was the one-step milling type. The flaky shape was still an important factor of the absorbing property, the previous study showed that the two-step milling process was proposed to control the milled FeSi particle size and shape [12], although the FeSi particle size was much larger than the CIPs, the milling process could be an potential way on changed the flake size. So, using the two-step milling process on the CIPs could be an effective way of enhancing the particle magnetic property. In addition, as the particles were added to the absorbing composite, the absorbing property of the single-layer absorber was limited due to the simple dispersion of the particles, while the multi-layer absorber could be an excellent structure on improving the absorbing property [13, 14]. The genetic algorithm might be an effective way on designing the multi-layer absorber, the CIPs/rubber absorbing composite with high absorption ratio could be obtained after the optimization 
process. Although the layer thickness and the layer number could be established, the volume content in the absorbing layer was only referring to the measured ones, the random volume content of each layer should be considered to improve the optimized result.

The present work is to investigate the microwave absorbing property of composites filled with two-step milled CIPs. First the flaky CIPs were fabricated using the two-step milling process. Secondly, the complex permittivity and permeability of CIPs composites were analyzed, and the optimized two-step milling process was selected. Finally the microwave absorbing property of the CIPs/rubber composite was optimized by simulating the RL in an application frequency using the genetic algorithm.

\section{Material and methods}

\subsection{Two-step milling on the CIPs}

The two-step milling on the CIPs included two parts, the first one was the high speed milling process on the particles, and the second one was the low speed milling process on the particles. The CIPs powders were introduced to a zirconia jar, and then a suitable analytically n-hexane, $\mathrm{ZrO}_{2}$ milling balls and stearic acid were added. The mass ratios of zirconia ball, CIPs, n-hexane and stearic acid were 20: 1: 1: 0.02. The $\mathrm{ZrO}_{2}$ milling balls included three different diameters, $20 \mathrm{~mm}, 8 \mathrm{~mm}$ and $5 \mathrm{~mm}$, the number of the three balls in one zirconia jar was 2, 50, and 500 respectively. The stearic acid, milling balls, n-hexane and mixed powders were sealed together in the zirconia jar, considering the n-hexane had the deoxidation effect and the CIPs oxidation could be neglected, it was not necessary to remove the atmosphere. The zirconia jar was fixed in the steel vial which was assembled to the SP2 planetary ball milling machine. The main axis in the planetary ball milling machine rotated with the velocity ratio of 100: 400 to the zirconia jar on its axis. As the milling time was selected, the zirconia jar was rotated at the high or low velocity respectively. After the milling process, the powders were sieved by a permanent magnet, washed using the ethanol to remove the stearic acid unbonded to the flaky CIPs, and then drilled in a baking oven.

The paper focused on the optimized milling time in the two-step milling process. The high speed milling velocity was set $500 \mathrm{r} / \mathrm{min}$ and the low speed milling velocity was selected as $250 \mathrm{r} / \mathrm{min}$. The high speed milling time was $1 \mathrm{~h}, 2 \mathrm{~h}, 3 \mathrm{~h}, 4 \mathrm{~h} 5 \mathrm{~h}$ or $6 \mathrm{~h}$, and the low speed milling time was $0 \mathrm{~h}, 5 \mathrm{~h}, 10 \mathrm{~h}, 15 \mathrm{~h}$ or 20h. The optimizing milling process on the CIPs was designed as shown in Fig.1. The time of the high speed milling and low speeding milling process was optimized in two steps. The first step was to optimize the low speed milling time, two high speeding milling times ( $1 \mathrm{~h}$ and $2 \mathrm{~h}$ ) were selected in order to 
decrease the optimized numbers, the particles were milled with velocity $500 \mathrm{r} / \mathrm{min}$ for $1 \mathrm{~h}$ or $2 \mathrm{~h}$ respectively, then the milled particles was milled with velocity $250 \mathrm{r} / \mathrm{min}$ for $0 \mathrm{~h}, 5 \mathrm{~h}, 10 \mathrm{~h}, 15 \mathrm{~h}$ and $20 \mathrm{~h}$. As a result, ten samples $(H i L j, i=1$ or 2 , and $j=0,5,10,15$ or 20 , the symbol $H i L j$ denotes the particle which was milled with high milling time $i \mathrm{~h}$ and low milling time $j \mathrm{~h}$, the other symbol could be explained similarly) could be obtained, and the ten kind particles were mixed into the rubber with volume content $50 \%$ respectively, the electromagnetic measurement sample could be fabricated. The permeability was selected to evaluate the optimized milling time, the sample with the high imaginary part of permeability could be considered as the optimized particles, then the optimized low-speeding milling time was established. The second step was to optimize the high speed milling time, as the optimized low milling time was set in the first step, the high speed milling time was selected as $1 \mathrm{~h}, 2 \mathrm{~h}, 3 \mathrm{~h}, 4 \mathrm{~h}, 5 \mathrm{~h}$ or $6 \mathrm{~h}$. Then several samples could be obtained and fabricated to evaluate the optimized high speed milling time. Finally, the optimized high and low speed milling time could be selected, and the optimized particle could be established, and the absorbing samples with variable volume content could be calculate to optimize the multilayer absorbing patch.

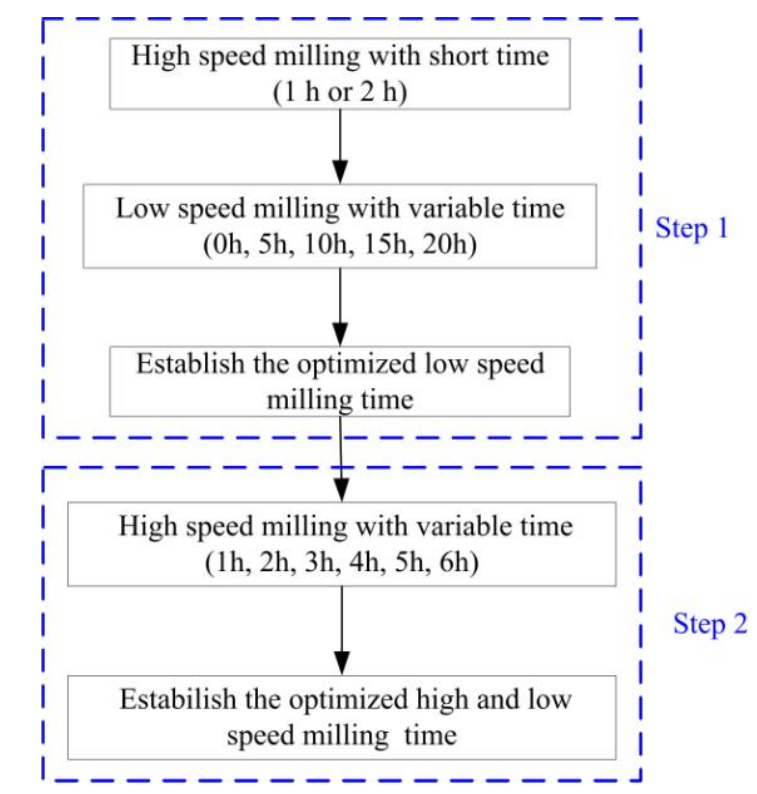

Fig. 1 The optimized process on the milling time using two steps

\subsection{Materials preparation and measurement}

Methyl vinyl Silicone rubber was used as matrix and 2, 5-dimethyl hexane was used as the vulcanized assistant, both were supplied by LaiZhou Jintai Silicon Industry Co. Ltd, China. Raw commercial flaky CIPs were supplied by Shanxi Xinghua Powder Co. Ltd, China. The average diameter 
of spherical CIPs was $3 \mu \mathrm{m}$. Samples filled with the single spherical or flaky CIPs with the volume content from $5 \%$ to $50 \%$ were fabricated respectively. The silicone rubber and absorbents were mixed in a two-roll mixer for 15-30 minutes. The particle was mixed into the silicone rubber with the uniform dispersion, for the roll-mixer provided a shear force in the mixing process which could overcome the intermolecular van der Waals force $[15,16]$. The testing samples for EM parameters measurement were modeled to a toroidal shape with outer diameter $7.0 \mathrm{~mm}$, inner diameter $3.04 \mathrm{~mm}$ and thickness $2 \mathrm{~mm}$. All the samples were vulcanized into pieces at $180^{\circ} \mathrm{C}$ under a pressure $10 \mathrm{MPa}$ for 5 minutes.

The morphology of the composites was observed by scanning electron microscopy (SEM CamScan CS3400) to evaluate the dispersion state and microstructure of CIPs. The effective complex permittivity and permeability of the RAM were measured using the AV3627 vector network analyzer in the frequency range of 2-18 GHz. The static magnetic property was then evaluated on a vibrating sample magnetometer (VSM, JDM-13), and the field reached up to $1.5 * 10^{4}$ Oe. The phase of powders was estimated by an X-ray diffractometer (D/MX 2200) using Cu K-radiation (wavelength $\lambda=0.154 \mathrm{~nm}$ ), and scan step size was 0.02 $\mathrm{deg} / \mathrm{s}$ with 50 steps per degree.

\subsection{Optimizing the RL using the genetic algorithm}

For a single-layer absorbing material, the RL of normal incident EM wave at the absorber surface is given by [17]:

$$
\begin{aligned}
& R L=20 \lg \left|\left(Z_{\text {in }}-Z_{0}\right) /\left(Z_{\text {in }}+Z_{0}\right)\right| \\
& Z_{\text {in }}=\sqrt{\mu_{r} \mu_{0} /\left(\varepsilon_{r} \varepsilon_{0}\right)} \tanh \left(j 2 \pi d \sqrt{\mu_{r} \varepsilon_{r}} / \lambda\right)
\end{aligned}
$$

where, $Z_{\text {in }}$ is the normalized input impedance of the absorber, $Z_{0}=\sqrt{\mu_{0} / \varepsilon_{0}}=120 \pi \Omega$ is the intrinsic impedance of free space, $\varepsilon_{r}, \mu_{r}$ and $\varepsilon_{0}, \mu_{0}$ are complex permittivity and complex permeability of the absorber and free space respectively, $\lambda$ is the wavelength, and $d$ is the thickness of the absorbing composites. While for the multi-layer absorbing plate, the input impedance of the $n$th layer $\left(Z_{\text {inn }}\right)$ is expressed as follows,

$$
\begin{aligned}
& Z_{i n n}=Z_{n} \frac{Z_{i n n-1}+Z_{n} \tanh \left(\gamma_{n} d_{n}\right)}{Z_{n}+Z_{i n n-1} \tanh \left(\gamma_{n} d_{n}\right)} \\
& Z_{i n 1}=Z_{1} \operatorname{th}\left(\gamma_{1} d_{1}\right)
\end{aligned}
$$


where, $Z_{n}=\sqrt{\mu_{n} / \varepsilon_{n}}$ is the characteristic impedance of the $n$th layer, $\varepsilon_{n}$ and $\mu_{n}$ was the permittivity and permeability of the $n$th layer, $\gamma_{n}=i 2 \pi f \sqrt{\varepsilon_{n} \mu_{n}} / C$ is the $n$th propagation constant, $d_{n}$ is the thickness of the $n$th layer, $C$ is the speed of the light.

So, the reflection coefficient $(R)$ and $R L$ of the absorber layer could be derived.

$$
\begin{aligned}
& R=\frac{Z_{i n n}-Z_{0}}{Z_{i n n}+Z_{0}} \\
& R L=20 \lg |R|
\end{aligned}
$$

The genetic algorithm was used to optimizing the layer characteristics and the thickness of the absorbing materials according to the RL requirement. Moreover, this paper proposed an interpolation process on the composite with the random volume content, as a result, the number of the selecting composite would increase, the optimized result could be enhanced. Firstly, the electromagnetic parameters library was built based on the testing composite added the same absorbent. The composites filled with the single absorbent with variable volume content were used to derive the composite with the random volume content. Then the electromagnetic parameters and the thickness of each layer were chosen. The chromosome of the genetic algorithm includes a sequence of bits, which contains information on each layer with its material number, volume content and thickness. $A_{i}, B_{j}$ and $C_{k}$ were defined to represent the material thickness, the material type and the volume content respectively, the sequence of bits for layer $n$ is like $A_{n} B_{n} C_{n}$, and the chromosome of the total layer can be expressed like $A_{1} B_{1} C_{1} A_{2} B_{2} C_{2} \cdots A_{m} B_{m} C_{m}$. So, the chromosome can be operated in the program. In the optimized process, the random population containing some chromosomes was created, then the number of material choice was decoded, the electromagnetic parameters could be used to calculate the fitness of each individual, then the select operation, cross operation and mutate operation was done. Finally, the optimized result could be obtained.

\section{Results and discussion}

\subsection{Characteristics of the particles}

The hysteresis loops for selected spherical CIPs and flaky CIPs under the applied field are shown in Fig.2. It showed that the loops curve had the typical characteristics of the soft magnetic particles, and the saturation magnetization (Ms) of the three particles was $200.34 \mathrm{emu} / \mathrm{g}$ and $195.02 \mathrm{emu} / \mathrm{g}$ respectively, and 
the Ms value of the CIPs was consistent with the results of other researches [17]. The Ms value of the flaky CIPs was decreased as the CIPs was changed to the flakes, but it was not much less than the value of spherical CIPs. It also indicated that the crystallization structure maybe change in the milling process, and the detailed result could be shown in the XRD result.

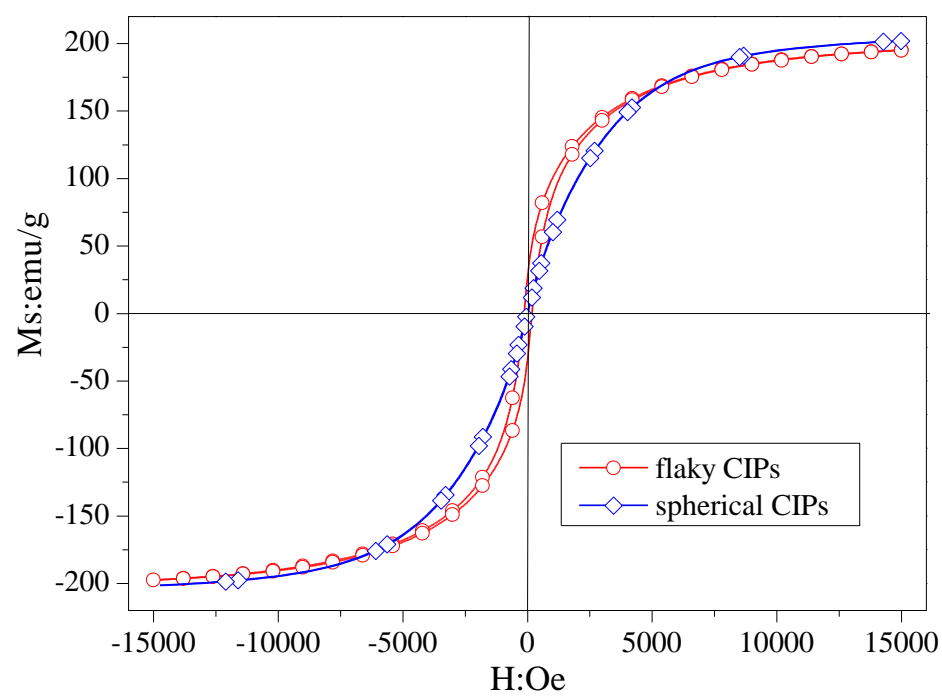

Fig.2 The hysteresis loops of the spherical and flaky CIPs

Fig.3 shows the X-ray diffraction patterns of the spherical CIPs, various milled flaky CIPs. The diffraction peaks of the single $\alpha$-Fe existed in the XRD pattern of CIPs, and the strength of the peaks is obvious. In XRD spectrum of the CIPs the peak at $2 \theta=44.5^{\circ}$ is the $\left(\begin{array}{lll}1 & 1 & 0\end{array}\right)$ plane, and the peak at $2 \theta=64.7^{\circ}$ is the $(200)$ plane. The XRD spectrum for the CIPs powder indicated the reflections corresponding to the distinct body-centered cubic (BCC) Fe metals. In addition, it was obtained that the characteristic peaks was broad and the intensity of the diffraction pattern was lower as the high-speeding milling time was $2 \mathrm{~h}$. It showed that the particle characteristics can be not only influenced by the milling parameters, but also by the crystallite size using the Scherrer's formula [10]. During the mechanical milling process, CIPs powders adhered on the $\mathrm{ZrO}_{2}$ balls, collision and milling occurred between the balls and the inner wall of the $\mathrm{ZrO}_{2}$ vial. The spherical powders would deform to smaller and flaky shape, and later the flaky particles were overlapped and the cold weld existed together, as a result, the flaky powders with various particle sizes were converted to the particle with uniform size. In the high speed milling process, the more energy was used, the particle tended to be milled to flakes easily. While in the low speed milling process, the particle collision happened especially for the cold welding particle. 


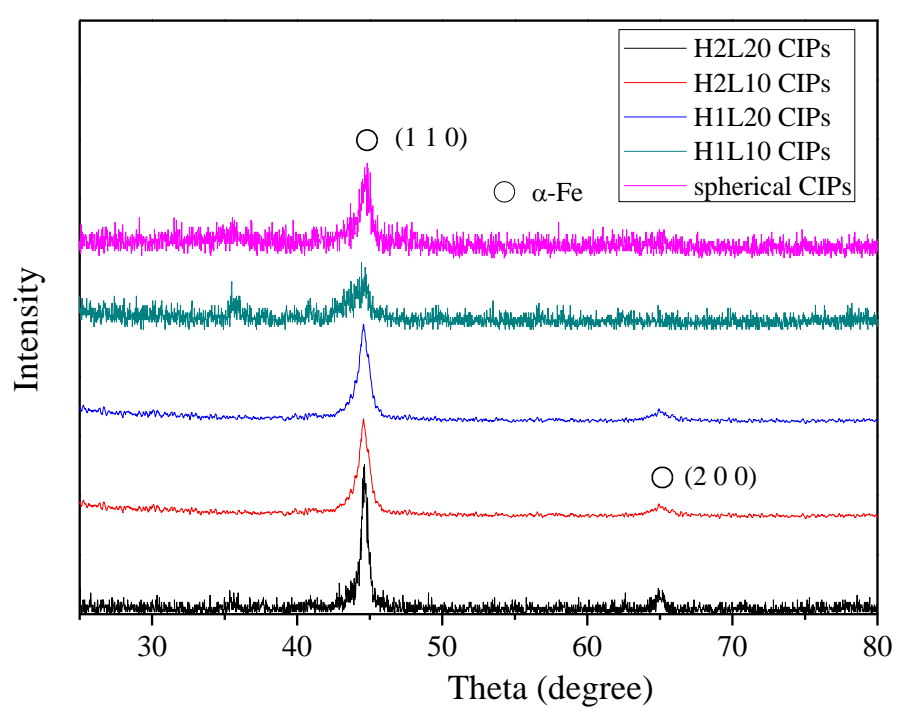

Fig.3 XRD patterns of the spherical and various flaky CIPs.

Fig.4 shows the energy spectrum analysis of the coating surface. It was observed that the atom of Fe and $\mathrm{C}$ existed on the surface of the flaky particle. The atom ratio of the flaky particle surface was $99.86 \%$ and $0.14 \%$. It could be obtained that the Fe atom mainly occupied a large atom content of the flaky particle, and the carbon still existed in the particle surface, while there was no $\mathrm{Zr}$ atom on the surface of the flaky particle surface. It was caused by the milling process, the high milling process had the velocity $500 \mathrm{r} / \mathrm{min}$, and the velocity was far less than the critical velocity $1000 \mathrm{r} / \mathrm{min}$. In addition, the milling process could transform the kinetic energy into the thermal energy, yet the added dispersing agent n-hexane made the heat dissipate, the alloying process seldom happened. The hardness of the milling ball $\mathrm{ZrO}_{2}$ was larger than that of CIPs, so was the milling jar, in the crash process the CIPs tended to collision, the $\mathrm{Zr}$ atom was seldom bonded to the flaky CIPs. As a result, the milling process mainly changed the particle shape, and the composition change in the flaky particle could be neglected in our milling process.

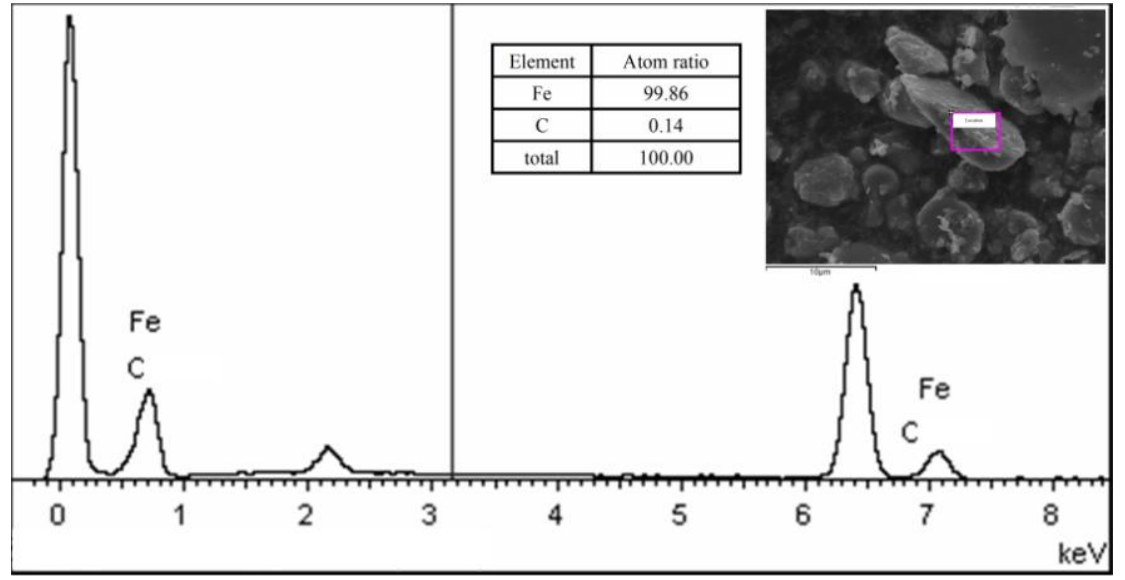

Fig.4 Energy spectrum analysis on the surface of the flaky particle. 


\subsection{Complex permeability of the flaky particle/rubber composites}

In the optimizing process for the low milling time, the high milling time was selected as $1 \mathrm{~h}$ or $2 \mathrm{~h}$. In the milling process, the permeability $(\mu)$ was the main factor for the flaky particle, for the permittivity of the milled particle often had a little influence on the absorbing property. So the sample which had the larger permeability was considered as the superior particle. Fig. 5 shows the the permeability of composites with the variable low milling time as a function of the frequency. As the volume content of the flaky CIPs was the same, the real part of permeability $\left(\mu^{\prime}\right)$ value decreased as the frequency increased in 2-18 GHz, the imaginary part of permeability $\left(\mu^{\prime \prime}\right)$ increased and then decreased in the frequency range. When the high milling time was set $1 \mathrm{~h}$ and the low milling time increased to $15 \mathrm{~h}, \mu^{\prime}$ was the maximum in $2-5 \mathrm{GHz}$ and $\mu^{\prime \prime}$ was the maximum in 2-8 GHz. While as the high milling time was set $2 \mathrm{~h}$, the sample with the low milling time $20 \mathrm{~h}$ had the maximum $\mu^{\prime \prime}$. For the magnetic loss materials the larger $\mu^{\prime \prime}$ often resulted in the better absorbing property with a thin thickness. So, the sample H2H20 could be seen as the most effective particle of the particles with variable low milling time. In addition, considering the low milling time $20 \mathrm{~h}$ might have a negative effect on improving the particle $\mu^{\prime \prime}$ compared with the sample H1L15 and H1L20. The optimized low milling time could be selected as $15 \mathrm{~h}$ and $20 \mathrm{~h}$, in order to optimize the high milling time, the variable high milling process was done as the low milling time was set.
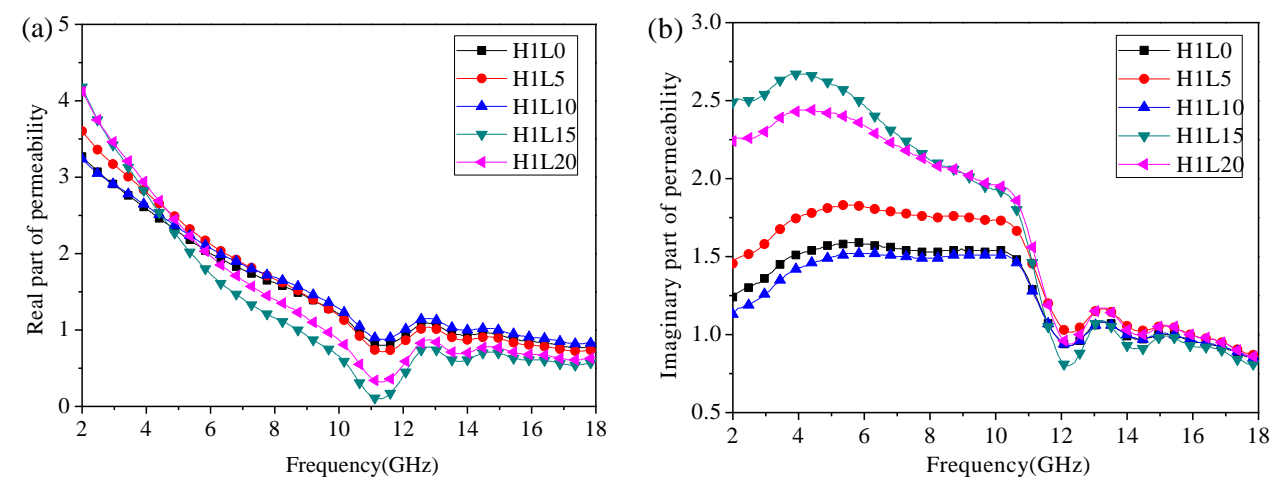

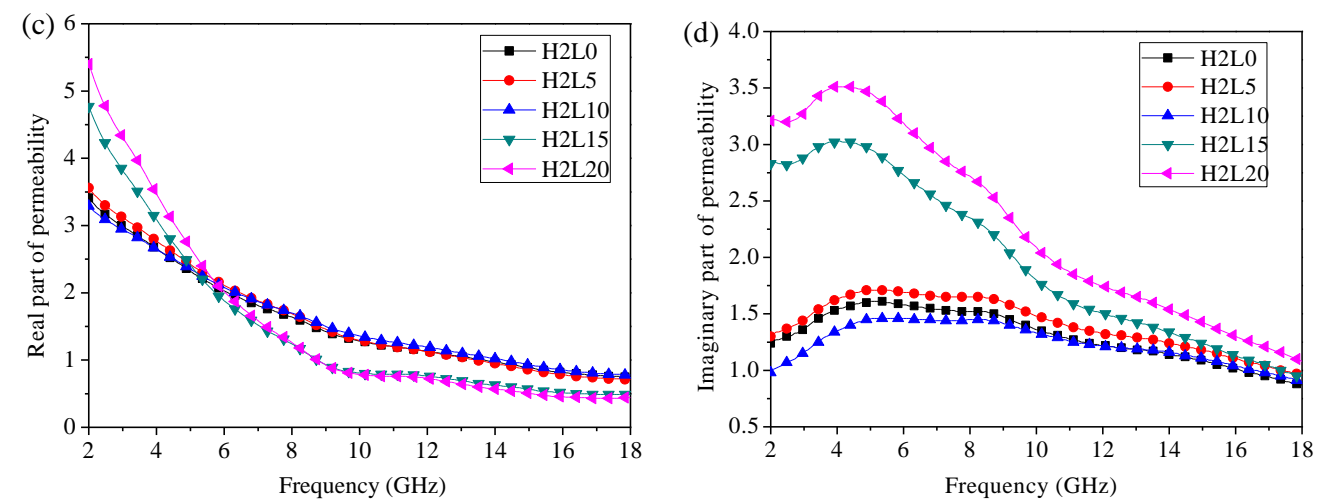

Fig. 5 The permeability of composites with variable low milling time, (a) the real permeability and (b) the imaginary permeability with high speed milling $1 \mathrm{~h}$, (c) the real permeability and (d) the imaginary permeability with high speed milling $2 \mathrm{~h}$

Fig. 6 shows the the permeability of composites with variable high milling time as a function of the frequency. As the low milling time was set, the high milling time was $2 \mathrm{~h}, 3 \mathrm{~h}, 4 \mathrm{~h}, 5 \mathrm{~h}$ or $6 \mathrm{~h}$. The high milling time $1 \mathrm{~h}$ was not included because the sample had a less $\mu^{\prime \prime}$ than other samples in the optimizing low milling process. As the low milling time was 15h, the sample H6L15 had the maximum $\mu^{\prime \prime}$ peak than other samples, the maximum $\mu^{\prime \prime}$ could be obtained at $4 \mathrm{GHz}$ and the value was about 3.6. While as the low milling time was $20 \mathrm{~h}$, it could be clearly obtained that the sample H2L20 had the maximum $\mu^{\prime \prime}$ than others. So, in order to optimize the high milling time, it could be obtained that the sample H6L15 and H2L20 had the largest permeability value, and the permeability of the two samples was very close. The high milling process was an energy-costing process, the particle could be broken and welded in the process, and the low milling process was mainly a broken process, the used energy could be less. The total milling time of the two samples was $21 \mathrm{~h}$ and $22 \mathrm{~h}$ respectively, therefore the milling energy on the sample H6L15 was much larger than the sample H2L20, the sample was considered as the optimized sample, and the optimized high milling time was $2 \mathrm{~h}$ and the low milling time was $20 \mathrm{~h}$.
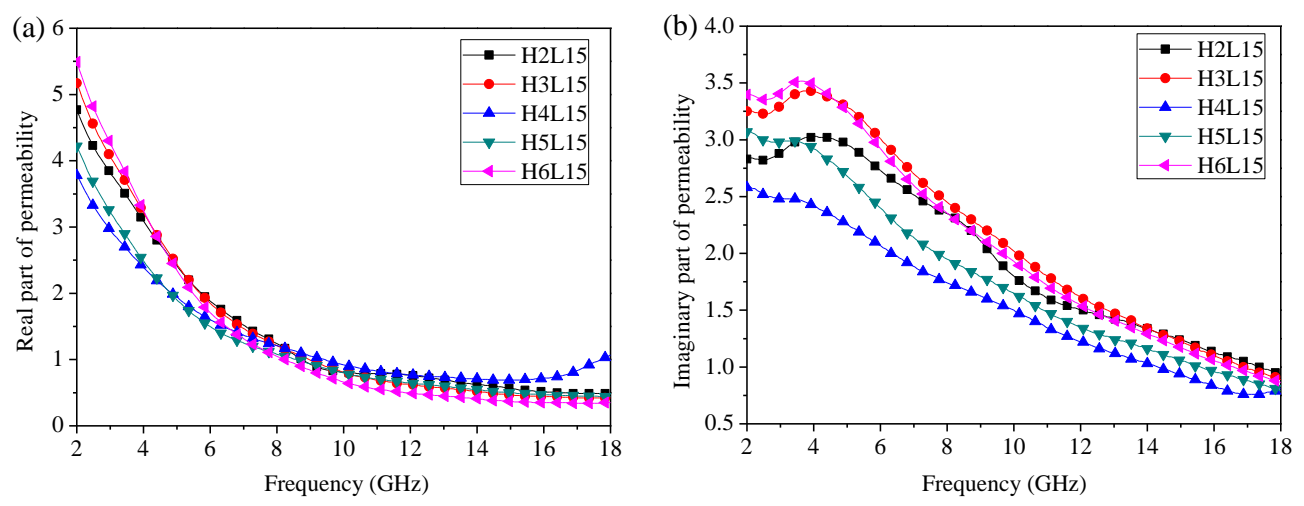

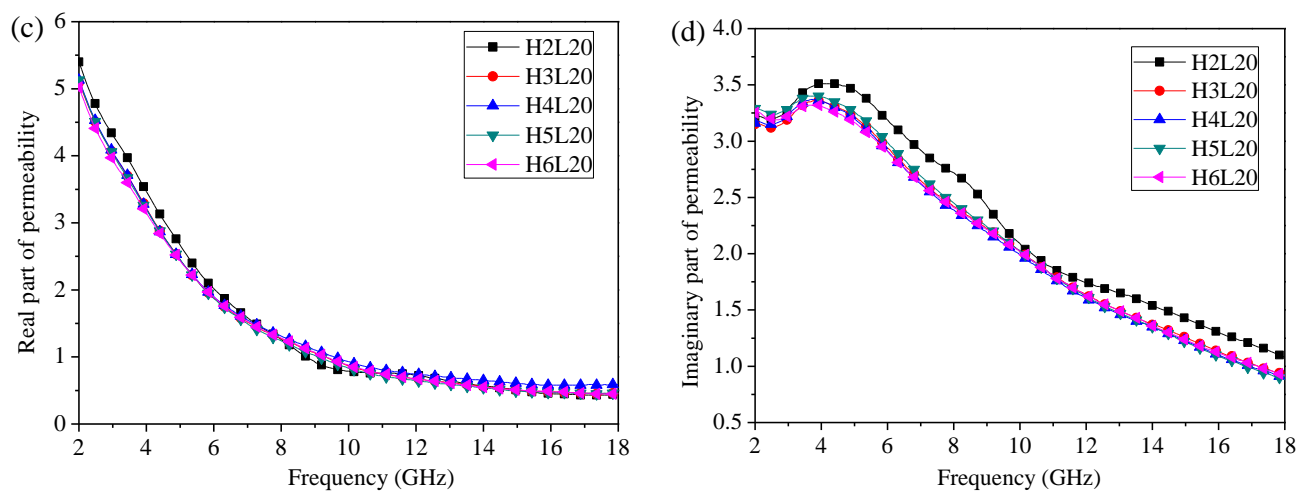

Fig. 6 The permeability of composites with variable high milling time, (a) the real permeability and (b) the imaginary permeability with low speed milling $15 \mathrm{~h}$, (c) the real permeability and (d) the imaginary permeability with low speed milling $20 \mathrm{~h}$

For the flaky magnetic particles, the permeability could be explained by the Maxwell-Garnett mixing rule. As the flaky particles were arbitrary oriented with a large volume content, the effective permittivity and permeability is isotropic and given as $[18,19]$ :

$$
\begin{aligned}
& \mu_{\text {eff }}=\mu_{m}+v \mu_{a} \frac{\mu_{i}-\mu_{m}}{\mu_{a}+\left(1-v / v_{c}\right) n\left(\mu_{i}-\mu_{m}\right)} \\
& \mu_{a}=\mu_{m}+a_{\mu}\left(\mu_{\text {eff }}-\mu_{m}\right)
\end{aligned}
$$

where $\mu_{i}$ and $\mu_{m}$ are the permeability of inclusion and matrix respectively, $\mu_{m}=1, \mu_{a}$ is the apparent permeability, $a_{\mu}$ is a pending parameter between 0 and $1, n$ is the averaged shape factor of the fillers, $n \approx 1 /\left(1+1.6 / e+0.4 / e^{2}\right)$ as the particle is flaky, where $1 / e$ is the aspect ratio, $v$ is the volume content and $v_{c}$ is the percolation threshold. For the magnetic materials, the intrinsic magnetic permeability $\mu_{i}$ of flaky CIPs can be obtained as $[18,19,20]$ :

$$
\mu_{i}=1+\frac{2}{3} \frac{\omega_{m}\left(\omega_{0}+i \alpha \omega\right)}{\left(\omega_{0}+i \alpha \omega\right)^{2}-\omega^{2}}
$$

where, $\omega_{m}=\gamma M_{s}, \omega_{0}=\gamma H_{a}, M_{s}$ is the saturation magnetization, $H_{a}$ is the magnetic anisotropy field, $\alpha$ is the damping coefficient, and $\gamma$ is the gyromagnetic ratio[21, 22]. The parameters in the above equation could be used to calculate the intrinsic permeability of the flaky CIPs, and then the effective permeability of the composite could be calculated using Maxwell-Garnett mixing rule. In order to obtain the effective permeability, the pending parameter including $h, v_{c}$ and $n$ could be established 
using a nonlinear least square interpolation method, it was used to calculate the intrinsic permeability according to the electromagnetic theory. Table 1 shows that the fitting parameters of the samples including $h, v_{c}$ and $n$, as a result, the aspect ratio $e$ could be calculated using the parameter $n$. The sample H2L20 had the most significantly flaky shape, the fitting aspect ratio $e$ was the biggest 14.64, which was much larger than the other samples. The sample H3L20 had the smallest aspect ratio 1.70, it indicated that the flaky shape was most unobvious.

Table 1 The fitting parameters of the samples

\begin{tabular}{ccccc}
\hline Sample & $h$ & $v_{c}$ & $n$ & $1 / e$ \\
\hline H2L20 & 0.5410 & 0.6078 & 0.0500 & 14.64 \\
H3L20 & 0.4593 & 0.5827 & 0.2595 & 1.70 \\
H4L20 & 0.3727 & 0.5719 & 0.1151 & 5.58 \\
H5L20 & 0.4168 & 0.5692 & 0.2286 & 2.12 \\
H6L20 & 0.4900 & 0.5957 & 0.1598 & 3.64 \\
H6L15 & 0.4576 & 0.5936 & 0.2018 & 2.59 \\
\hline
\end{tabular}

Fig. 7 shows the morphology of the milled particles. It could be obtained that all the milled samples were of the flaky shape, the particle size of the samples was analyzed using the microscopic method. The microscopic method was a useful method to analyze the particle size by the statistic calculation using the SEM images. The right top area in each figure was selected as the calculation zone. The average diameters of the six samples were 8.64, 5.90, 7.52, 6.21, 8.12 and 7.55 respectively. The sample H2L20 had the maximum particle size (average $8.64 \mu \mathrm{m}$ ), much bigger than other samples, the sample H3L20 had the smallest particle size, the average diameter was only $5.90 \mu \mathrm{m}$. According to the fitted aspect ratio, the thickness of the two particles was about $0.59 \mu \mathrm{m}$ and $3.34 \mu \mathrm{m}$. It could be obtained that as the low milling time increased from $15 \mathrm{~h}$ to 20 , the sample diameter might increase compared with the sample H6L20 and H6L15. While adding the high milling time the milled particle diameter did not increase gradually among the samples with the high milling time $20 \mathrm{~h}$, there might be an interaction on the high milling process and the low milling process, the detailed mechanism would be demonstrated in a further research. Moreover, compared with the imaginary permeability of the six samples, the particle size affected the permeability, the permeability of the samples with the larger aspect ratio could have a larger imaginary permeability, and with the smaller diameter the permeability of the samples was also smaller, which was consistent to the previous research [10,22]. 

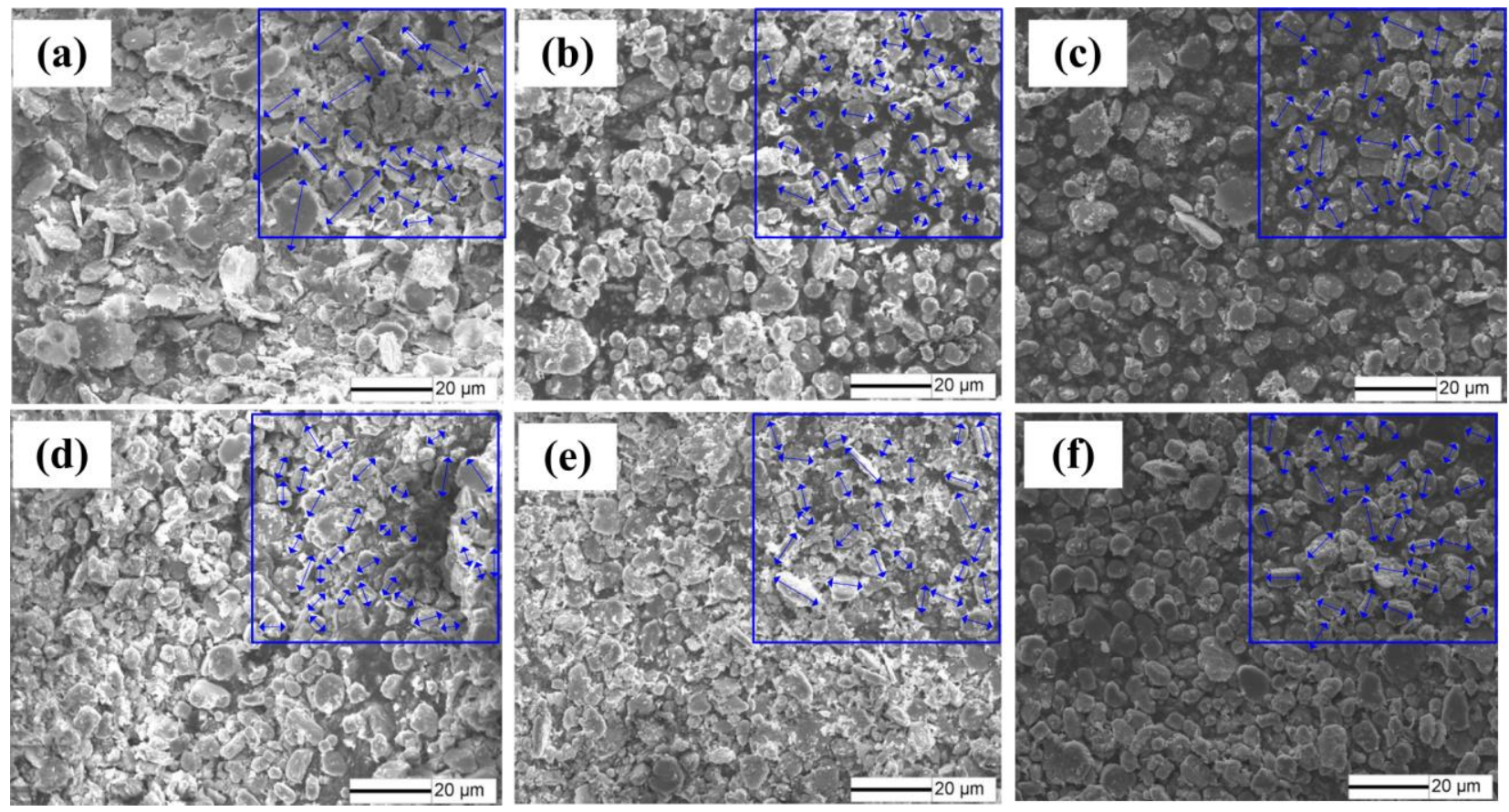

Fig. 7 Morphology of the flaky particles. (a) H2L20, (b) H3L20, (c) H4L20, (d) H5L20, (e) H6L20, (f) H6L15.

\subsection{Optimizing the RL of the CIPs/rubber composites}

In order to optimizing the absorbing property of the CIPs/rubber composite using the genetic algorithm, the particle of the spherical and the flaky shape with variable volume content were both selected. The volume content of the spherical particle was $0 \%, 10 \%, 20 \%, 30 \%$, or $40 \%$, the volume content of the flaky particle was $10 \%, 20 \%, 30 \%, 40 \%$ or $45 \%$. The random volume content of the absorbing materials was obtained using the Hermite interpolation method based on the testing results of composites added the single spherical CIPs or flaky CIPs. In the Hermite interpolation process, the scattering parameters $S_{11}$ and $S_{12}$ was defined as the function of the absorbent volume content. According to the Nicolson-Ross-Weir algorithm [23],

$$
\begin{gathered}
S_{11}=\frac{\left(1-T^{2}\right) \Gamma_{0}}{1-T^{2} \Gamma_{0}} \\
S_{21}=\frac{\left(1-\Gamma_{0}^{2}\right) T}{1-T^{2} \Gamma_{0}^{2}}
\end{gathered}
$$

$\Gamma_{0}$ is the reflection coefficient of the material surface, $\Gamma_{0}=\left(\sqrt{\mu_{r} / \varepsilon_{r}}-1\right) /\left(\sqrt{\mu_{r} / \varepsilon_{r}}+1\right), T$ was the transition coefficient in the materials, $d$ was the material thickness, $\gamma$ was the propagation constant, $\gamma=2 \pi \sqrt{\mu_{r} \varepsilon_{r}} / \lambda_{0}$. So, using the tested electromagnetic parameters ( $\varepsilon$ and $\mu$ ), the two scattering parameters could be established using the above algorithm as the material thickness was set constant 2 $\mathrm{mm}$. Then the Hermite interpolation was done, considering that the function value of $n+1$ mutually exclusive points $x_{0}, x_{1}, x_{2}, \cdots, x_{n}$ were $y_{0}, y_{1}, y_{2}, \cdots, y_{n}$, and the derivative values of $m+1$ points in the 
$n+1$ ones are $y_{i 0}, y_{i 1}, y_{i 2}, \cdots, y_{i m}$, then the polynomial function could be obtained,

$$
H(x)=\sum_{k=0}^{m+n+1} a_{k} x_{k}
$$

where the following equations could be satisfied,

$$
\left\{\begin{array}{l}
H\left(x_{i}\right)=y_{i} \quad(i=0,1, \ldots, n) \\
H^{\prime}\left(x_{i k}\right)=y_{i k}^{\prime} \quad(k=0,1, \ldots, m)
\end{array}\right.
$$

The pivotal of Hermite method used for the interpolation of the scattering parameters is to solve the derivative of the scattering parameters for individual volume fraction. Then the permittivity $\left(\varepsilon_{r}\right)$ and the permeability $\left(\mu_{r}\right)$ could be deduced,

$$
\begin{aligned}
& T=\left[S_{11}+S_{21}-\Gamma_{0}\right] /\left[1-\left(S_{11}+S_{21}\right) \Gamma_{0}\right] \\
& \Gamma_{0}=K \pm \sqrt{K^{2}-1}
\end{aligned}
$$

Where, $K=\left[\left(S_{11}^{2}-S_{12}^{2}\right)+1\right] /\left(2 S_{11}\right)$, and according to the above equations, $\varepsilon_{r}$ and $\mu_{r}$ could be observed.

$$
\begin{aligned}
& \varepsilon_{r}=\frac{\left(\frac{1}{\Lambda^{2}}+\frac{1}{\lambda_{c}^{2}}\right) \lambda_{0}^{2}}{\mu_{r}} \\
& \mu_{r}=\left(1+\Gamma_{0}\right) /\left[\Lambda\left(1-\Gamma_{0}\right) \sqrt{\frac{1}{\lambda_{0}^{2}}-\frac{1}{\lambda_{c}^{2}}}\right]
\end{aligned}
$$

where, $\Lambda^{2}=-[\ln (T) / 2 \pi d]^{-2}, \quad \lambda_{0}$ is the microwave wavelength in the air, and $d$ is the thickness of the absorbing material, $\lambda_{c}$ is the cutoff wavelength of TEM.

The permittivity and permeability of the absorbing materials were showed in Fig. 8 and Fig. 9 as a function of the frequency. It could be obtained that as the volume content increased, the permittivity and the permeability was both increased. As the volume concentration was the same, the real part of permittivity $\left(\varepsilon^{\prime}\right)$, the real part of permeability $\left(\mu^{\prime}\right)$ and the imaginary part of permeability $\left(\mu^{\prime \prime}\right)$ of the composites added the flaky particle are much larger than that of the spherical composite respectively, it was owing to the particle shape in the micrometer size, which is consistent with the previous study $[6,10]$. In addition, using the Hermite interpolation on the composite, the composite added $30 \%$ spherical CIPs or $40 \%$ flaky CIPs, the calculated result was in good accordance with the measured one, the average permittivity deviation of two composites were $0.39+\mathrm{j} 0.45$ and $2.5+\mathrm{j} 0.51$, and the average permeability deviation were $0.03+\mathrm{j} 0.09$ and $0.03+\mathrm{j} 0.09$, respectively. The interpolation result showed that the 
permeability deviation was much smaller than the permittivity deviation, which indicated that the interpolation algorithm was very effective to calculate the composite absorbing property, because the RL was often mainly determined by the complex permeability for the CIPs composites.
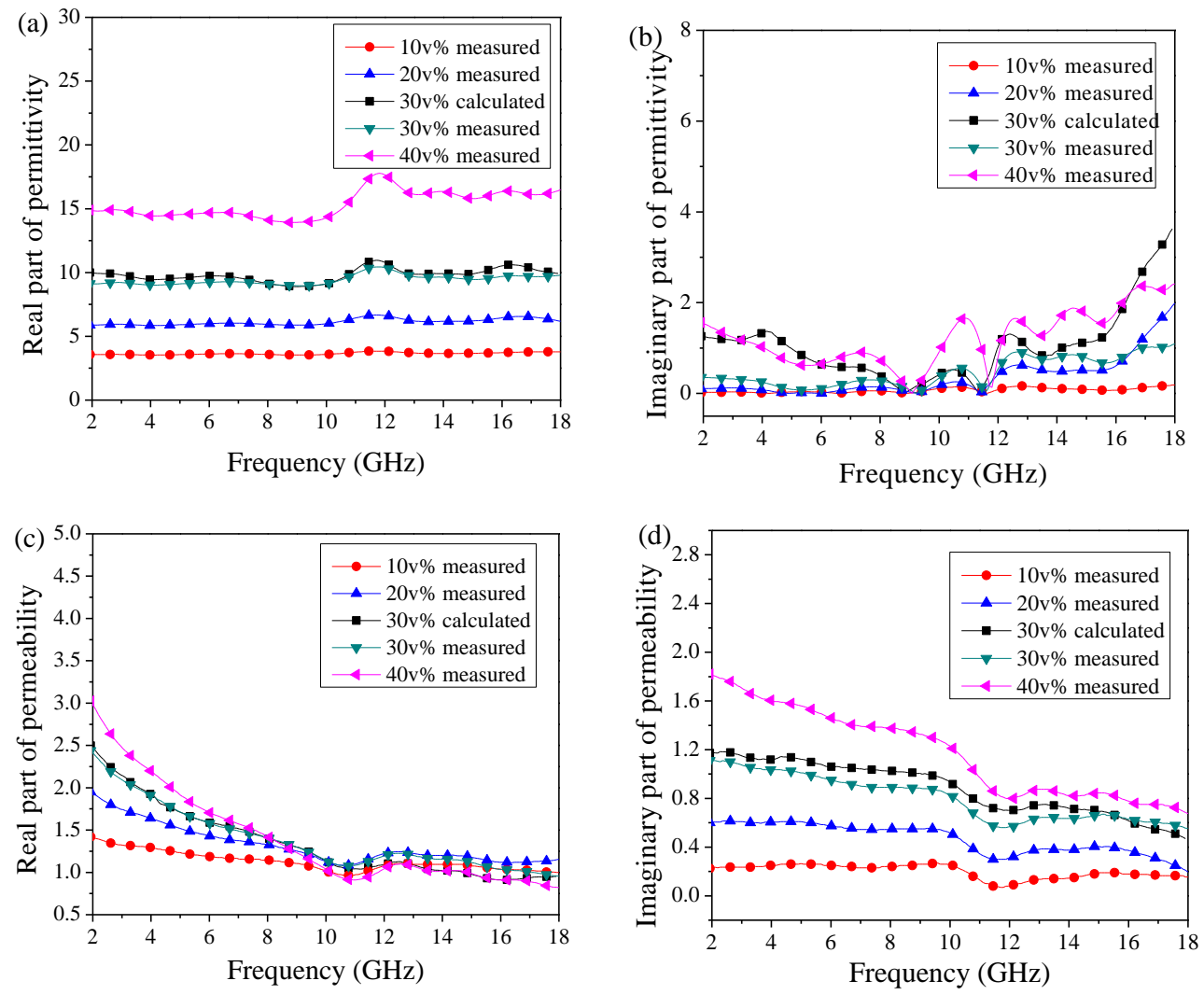

Fig. 8 EM parameters of spherical CIPs with variable volume content, (a) the real permittivity, (b) the imaginary permittivity, (c) the real permeability, (d) the imaginary permeability
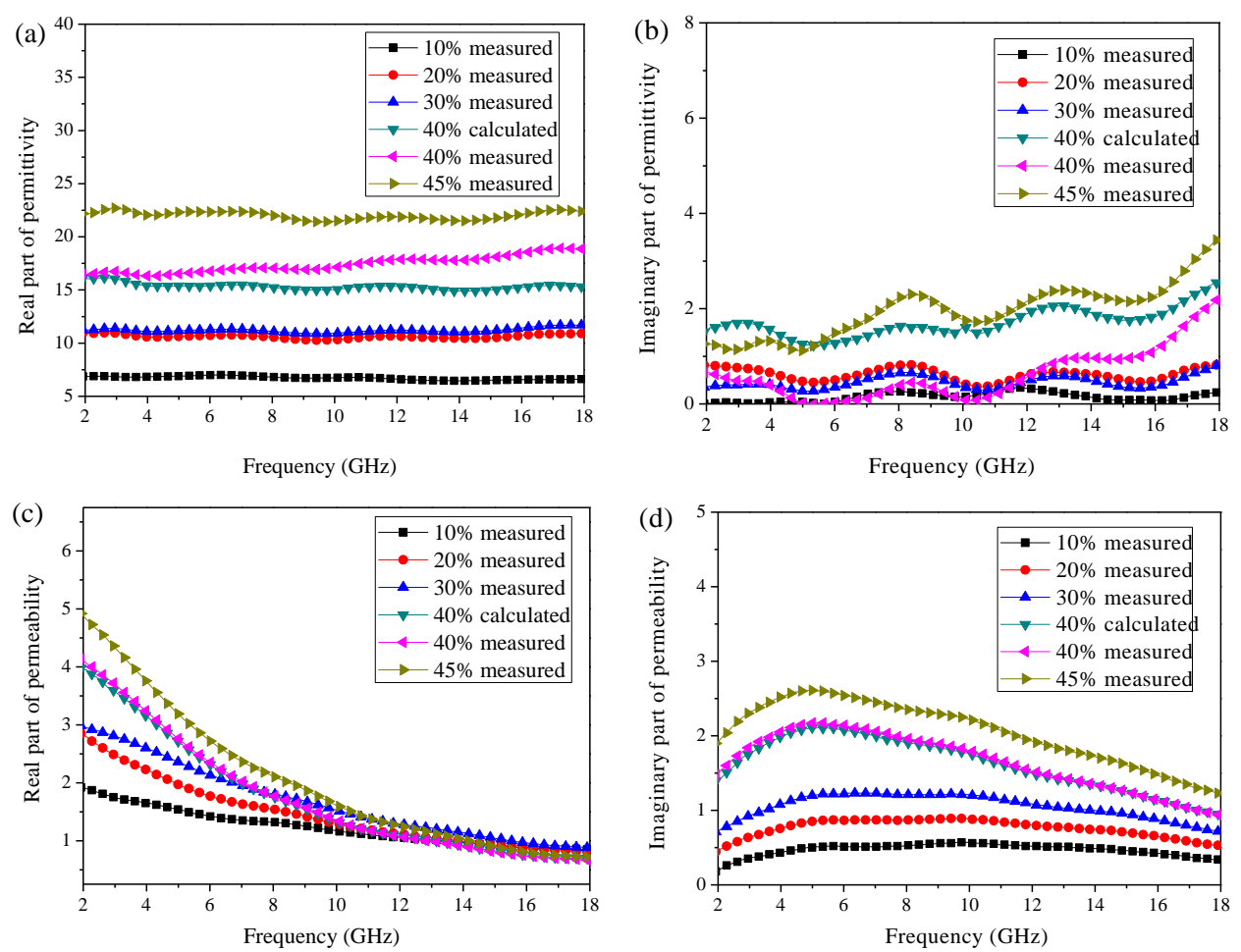
Fig. 9 EM parameters of flaky CIPs with variable volume content, (a) the real permittivity, (b) the imaginary permittivity, (c) the real permeability, (d) the imaginary permeability

Using the above materials, the genetic algorithm based on the interpolation process was set to optimize the RL in frequency $8-18 \mathrm{GHz}$, the RL lower than $-10 \mathrm{~dB}$ was required, and the thickness should be thin as much as possible. The cross coefficient was 0.9 , the initial population number was 1000 and the maximum generated number was 15 . Considering the mutation coefficient was an important factor in the genetic algorithm, the value was set as $0.04,0.05,0.06,0.07$ or 0.08 in the optimizing process, as a result, the optimized RL could be obtained.

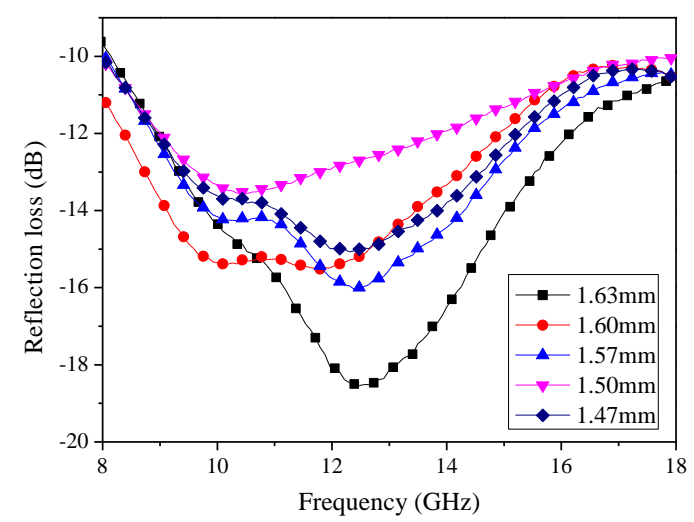

Fig. 10 The optimized RL of the composite with variable thickness

Fig. 10 showed the optimized RL with variable thickness and mutation coefficient as a function of frequency. All the RL was lower than $-10 \mathrm{~dB}$, which meant that the absorbing coefficient on the microwave energy was more than $90 \%$. As the optimized thickness decreased, the minimum RL gradually increased from $-17.18 \mathrm{~dB}$ at thickness $1.63 \mathrm{~mm}$ to $-13.56 \mathrm{~dB}$ at thickness $1.5 \mathrm{~mm}$. It indicated that the absorbing property could be enhanced as the composite thickness increased. The component of the optimized composite was showed in Table 2. In the optimized composite, the layer number was 3 or 4. The symbol for the material type F-42\% denotes the flaky CIPs composite with volume content $42 \%$, while the material type F-22\% denotes the flaky CIPs with volume content $22 \%$, the others could be explained similarly. Among the optimized five composites, the obtained thickness decreased as the mutation coefficient increased, the minimum thickness was $1.47 \mathrm{~mm}$. In addition, the S-5\% sample was used in the composite with thickness $1.50 \mathrm{~mm}$, which meant that the matching space layer added a few absorbent was a part of the composite. Although the thickness was larger than the composite with thickness $1.47 \mathrm{~mm}$, the surface density of the composite would decrease as matching space layer added, the calculated surface density was $4.10 \mathrm{~kg} / \mathrm{m}^{2}$ and $4.70 \mathrm{~kg} / \mathrm{m}^{2}$ respectively.

Table 2 Design on the RL of composite using the genetic algorithm 


\begin{tabular}{ccccccc}
\hline No. & $\begin{array}{c}\text { 1st layer } \\
\text { Thickness(mm), } \\
\text { Type-volume content }\end{array}$ & $\begin{array}{c}\text { Thickness(mm), } \\
\text { Type-content }\end{array}$ & $\begin{array}{c}\text { 3rd layer } \\
\text { Thickness(mm), } \\
\text { Type-volume content }\end{array}$ & $\begin{array}{c}\text { 4th layer } \\
\text { Thickness(mm), } \\
\text { Type-volume } \\
\text { content }\end{array}$ & $\begin{array}{c}\text { Total } \\
\text { thickness } \\
(\mathrm{mm})\end{array}$ & $\begin{array}{c}\text { Mutation } \\
\text { coefficient }\end{array}$ \\
\hline 1 & $0.47, \mathrm{~F}-38 \%$ & $0.16, \mathrm{~S}-5 \%$ & $0.50, \mathrm{~S}-18 \%$ & $0.50, \mathrm{~F}-22 \%$ & 1.63 & 0.04 \\
2 & $0.40, \mathrm{~F}-43 \%$ & $0.77, \mathrm{~S}-19 \%$ & $0.43, \mathrm{~F}-21 \%$ & ----- & 1.60 & 0.05 \\
3 & $0.40, \mathrm{~F}-44 \%$ & $0.43, \mathrm{~S}-22 \%$ & $0.50, \mathrm{~S}-12 \%$ & $0.23, \mathrm{~F}-39 \%$ & 1.56 & 0.06 \\
4 & $0.30, \mathrm{~F}-44 \%$ & $0.30, \mathrm{~F}-39 \%$ & $0.40, \mathrm{~S}-4 \%$ & $0.50, \mathrm{~F}-19 \%$ & 1.50 & 0.07 \\
5 & $0.47, \mathrm{~F}-42 \%$ & $0.67, \mathrm{~S}-18 \%$ & $0.33, \mathrm{~F}-42 \%$ & ----- & 1.47 & 0.08 \\
\hline
\end{tabular}

Oftenly, the good absorbing material should satisfy two important conditions, the matching characteristics and the attenuation characteristics. The composite filled with CIPs had the larger dielectric loss tangent and magnetic loss tangent in the frequency range of 2-18 GHz. So in order to achieve the good absorbing property the designed composite could satisfy the two requirements as far as possible, the gradient property should be designed. In this paper, only the layer-type absorbing composite was studied, yet the particle dispersion density and structure also affected the absorbing property of the absorbing materials, so the internal structure of the composite could be considered in the further research.

\section{Conclusion}

The two-step milling process could enhance the permeability of the flaky CIPs composite related to the one step milling particles. The low speeding milling process on the flaky CIPs was important on breaking the flaky particles, in which the particle aspect ratio could increase. The Hermite interpolation using the scattering parameters of the composites was effective to derive the permittivity and permeability of the composite with random volume content. The genetic algorithm based on the parameter interpolating was effective to optimize the absorbing property of the composite in 8-18 GHz. The RL value was lower than $-10 \mathrm{~dB}$ with the optimized thickness $1.47 \mathrm{~mm}$, and the surface density decreased as the optimized thickness was $1.50 \mathrm{~mm}$.

\section{Acknowledgement}

This work was supported by the Program of Shanghai Subject Chief Scientist (B type) (Grant No. 14XD1423100), the National Natural Science Foundation of China (Grant No. 61471242, No. 61302036), and the National Science Foundation for The Excellent Youth Scholars of Ministry of Education of China (Grant No. 51322503). 


\section{References}

[1] Micheli D, Apollo C, Pastore R, Marchetti M. X-Band microwave characterization of carbon-based nanocomposite material, absorbing capability comparison and RAS design simulation. Compos Sci Technol 2010; 70(2): 400-9

[2] Sang-Eui Lee, Oyoung Choi, Thomas H. Hahn. Microwave properties of graphite nanoplatelet/epoxy composites. J Appl Phys 2008; 104: 033705.

[3] Itoh M, Liu J, Horikawa T, Machida K-I. Electromagnetic wave absorbing properties of nanocomposite powders derived from intermetallic compounds and amorphous carbon. J Alloys Compd 2006; 408: 1400-3.

[4] Snoek JL. Dispersion and absorbing in magnetic ferrites at frequencies above one Mc/s. Physica 1948; 14 (4): 207-17.

[5] Lebourgeois R, Fur CL, Labeyrie M, Pate M, Ganne JP. Permeability mechanisms in high frequency polycrystalline ferrites. J Magn Magn Mater 1996; 160(1): 329-32.

[6] Zhang BS, Feng Y, Xiong J, Yang Y, Lu HX. Microwave-absorbing properties of de-aggregated flake-shaped carbonyl-iron particle composites at 2-18 GHz. IEEE Trans Magn 2006; 42(7): 1778-81.

[7] Liu LD, Duan YP, Liu SH, Chen LY, Guo JB. Microwave absorbing properties of one thin sheet employing carbonyl-iron powder and chlorinated polyethylene. J Magn Magn Mater 2010; 322: $1736-40$.

[8] Gilbert TL. A phenomenological theory of damping in ferromagnetic materials. IEEE Trans. Magn. 2004; 40(6): $3443-9$.

[9] Desheng Xue, Fasheng Li, Xiaolong Fan, Fusheng Wen. Bianisotropy picture of higher permeability at higher frequencies. Chin Phys Lett 2008; 25(11): 4120-3.

[10] Fusheng Wen, Wenliang Zuo, Haibo Yi, Nan Wang, Liang Qiao, Fashen Li. Microwave-absorbing properties of shape-optimized carbonyl iron particles with maximum microwave permeability. Physica B: Condensed Matter 2009. 404(20), 3567-70.

[11] Jun Liu, Yongbao Feng, Tai Qiu. Synthesis, characterization, and microwave absorption properties of $\mathrm{Fe}-40 \mathrm{wt} \% \mathrm{Ni}$ alloy prepared by mechanical alloying and annealing. Journal of Magnetism and Magnetic Materials. 2011, 323(23): 3071-76. 
[12] Hualiang Lv, Guangbin Ji, Xiaoguang Li, Xiaofeng Chang, Min Wang, Haiqian Zhang, Du Youwei. Microwave absorbing properties and enhanced infrared reflectance of $\mathrm{FeAl}$ mixture synthesized by two-step ball-milling method. Journal of Magnetism and Magnetic Materials 2015. 374: 225-9.

[13] Yuan Jie, Xiao Gang, Cao Mao-Sheng. A novel method of computation and optimization for multi-layered radar absorbing coatings using open source software. Materials and Design (2006). 27: 45-52.

[14] Sotirios K. Goudos. A versatile software tool for microwave planar radar absorbing materials design using global optimization algorithms. Materials and Design (2007). 28: 2585-95.

[15] Sandler J, Shaffer M, Prasse T, Bauhofer W, Schulte K, Windle A.H. Development of a dispersion process for carbon nanotubes in an epoxy matrix and the resulting electrical properties. Polymer 1999; 40(21): 5967-71.

[16] Iosif D. Rosca, Suong V. Hoa. Highly conductive multiwall carbon nanotube and epoxy composites produced by three-roll milling. Carbon 2009; 47(8): 1958-68.

[17] Rui Han, Jianqiang Wei, Xianghua Han, Haibo Yi, Tao Wang, Fashen Li. High-frequency magnetic properties of carbonyl-iron particles/paraffin composite [J]. Chinese Science Bulletin, 2010, 55(26): 2570-5

[18] J.L. Wallace. Broadband magnetic microwave absorbers: Fundamental Limitations. IEEE Trans. Magn. 1993, 29 (6): 4209-14.

[19] P. Chen, M. L., L. Wang, Y. Poo, R.X. Wu. Frequency dispersive complex permittivity and permeability of ferromagnetic metallic granular composite at microwave frequencies. J. Magn. Magn. Mater. 2011, 323: 3081-6.

[20] P. Chen, R.X. Wu, T.E. Zhao, F. Yang, John Q Xiao. Complex permittivity and permeability of metallic magnetic granular composites at microwave frequencies. Journal of Physics D: Applied Physics. 2005, 38: 2302-5

[21] T. Liu, P.H. Zhou, J.L. Xie, L.J. Deng. Extrinsic permeability of Fe-based flake composites from intrinsic parameters: A comparison between the aligned and random cases. J. Magn. Magn. Mater. 2012, 324(4): 519-23.

[22] L.Z. Wu, J. Ding, H.B. Jiang, L.F. Chen, C.K. Ong. Particle size influence to the microwave properties of iron based magnetic particulate composites. J. Magn. Magn. Mater. 2005, 285(1-2): 233-9. 
[23] De Paula, A.L., Barroso, J.J., Rezende M.C. Modified Nicolson-Ross-Weir (NRW) method to retrieve the constitutive parameters of low-loss materials. Microwave \& Optoelectronics Conference.2011, 488-92. 


\section{Figure and table captions:}

Fig. 1 The optimized process on the milling time using two steps

Fig.2 The hysteresis loops of the spherical and flaky CIPs

Fig.3 XRD patterns of the spherical and various flaky CIPs.

Fig. 4 Energy spectrum analysis on the surface of the flaky particle.

Fig. 5 The permeability of composites with variable low milling time, (a) the real permeability and (b) the imaginary permeability with high speed milling $1 \mathrm{~h}$, (c) the real permeability and (d) the imaginary permeability with high speed milling $2 \mathrm{~h}$.

Fig. 6 The permeability of composites with variable high milling time, (a) the real permeability and (b) the imaginary permeability with low speed milling $15 \mathrm{~h}$, (c) the real permeability and (d) the imaginary permeability with low speed milling $20 \mathrm{~h}$.

Fig. 7 Morphology of the flaky particles. (a) H2L20, (b) H3L20, (c) H4L20, (d) H5L20, (e) H6L20, (f) H6L15.

Fig. 8 EM parameters of spherical CIPs with variable content, (a) real permittivity, (b) imaginary permittivity, (c) the real permeability, (d) imaginary permeability.

Fig. 9 EM parameters of flaky CIPs with variable content, (a) real permittivity, (b) imaginary permittivity, (c) the real permeability, (d) imaginary permeability.

Fig. 10 The optimized RL of the composite with variable thickness.

Table 1 The fitting parameters of the samples.

Table 2 Design on the RL of composite using the genetic algorithm. 


\section{Graphical abstract}

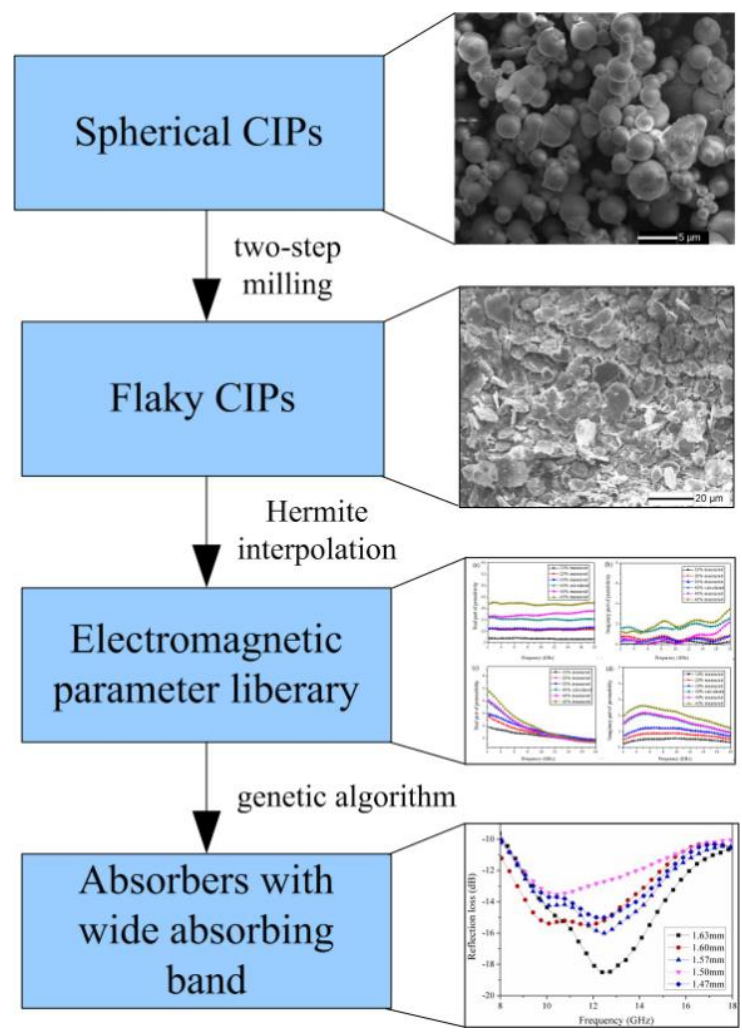

Fig.1 The property of absorber added two speeding milling CIPs could be enhanced using the genetic algorithm. 